\section{CAMBIO CULTURAL Y CONTACTO EN LA PREHISTORIA DE LA EUROPA MEDITERRANEA (1)}

\author{
CONTACT AND CULTURE CHANGE IN \\ THE PREHISTORY OF MEDITERRANEAN \\ EUROPE
}

\section{ANTONIO GILMAN GUILLEN ( $)$}

\section{RESUMEN}

La importancia del comercio maritimo en épocas históricas ha servido como modelo para la interpretación de la prehistoria mediterránea. Para los normativistas que prevalecieron hasta recientemente, cualquier parecido particular entre conjuntos arqueológicos podría ser prueba de un comercio de ideas. Al restringir el término comercio a intercambios cuantificables, los procesualistas han sostenido que el desarrollo prehistórico de cada región del Mediterráneo era autónomo, un punto de vista quizás demasiado limitado. Los enfoques de tipo sistema mundial brindan la oportunidad de dar un peso procesual a los contactos entre las sociedades, pero sólo funcionan cuando hay una valoración adecuada de la estructura social y económica tanto del centro como de la periferia.

\begin{abstract}
The historically documented importance of seafaring commerce has served as a charter for the interpretation of Mediterranean prehistory. Within the normativist framework that prevailed until recently, any similarity in the particulars
\end{abstract}

(1) Una versión preliminar de este artículo fue presentada a la 25 Conferencia Chacmool en Calgary (Alberta, Canadá) en noviembre de 1992. El texto que aquí se publica ha sido traducido por M. ${ }^{\mathrm{a}}$ I. Martínez Navarrete y revisado por el autor.

(*) Department of Anthropology. California State University. Northridge. CA 91330. of archaelogical assemblages could be proof of a commerce of ideas. By limiting the notion of commerce to quantifiable exchanges, processualists have argued for the autonomous development of each region within the prehistoric Mediterranean, a perhaps excessively restricted view. World-systems approaches offer the opportunity to give processual weight to intersocietal contacts, but only are successful when the social and economic structure of both center and periphery is adequately understood.

Palabras clave: Contacto. Comercio. Mediterráneo. Sistenıa Mundial. Hierro. Bronce.

Key words: Contact. Commerce. Mediterranean. Worldsystem. Iron. Bronze.

\section{I}

Cuando la historia comienza en la Europa mediterránea, lo hace como resultado directo de un "contacto": los griegos han desarrollado su alfabeto a partir de modelos fenicios. El fuerte paralelismo en el desenvolvimiento de los asentamientos urbanos en Grecia e Italia durante el s. VIII y en España durante el s. VII tiene lugar bajo auspicios orientalizantes. Estos florecimientos del inicio de la Edad del Hierro 
implicaron un proceso en el cual la importación de bienes y la emulación de estilos de origen fenicio y egipcio (a través de intermediarios fenicios) jugaron una no pequeña parte. A comienzos del Primer Milenio a. C. el comercio marítimo de bienes e ideas estaba tan en funcionamiento que convertiría al Mediterráneo en el centro del mundo occidental civilizado hasta mediados del Segundo Milenio A. D. No es de extrañar, por tanto, que los prehistoriadores del mundo mediterráneo usaran la importancia históricamente documentada de contacto como un modelo con el cual explicar la variabilidad de la evidencia del Neolítico, la Eaad del Cobre y la Edad del Bronce. Se asumía que los cambios en el registro arqueológico de una región particular eran el resultado de la llegada de nuevos elementos del exterior. Incluso un rápido examen de las ilustraciones de las publicaciones sobre yacimientos arqueológicos de otras regiones proporcionaría los paralelos requeridos para los materiales propios.

Cuanto más al occidente del Mediterráneo trabajara uno, más amplio era el abanico geográfico de los paralelos que uno podía trazar. Aquéllos de nosotros que nos ocupamos de la Península Ibérica somos perfectamente conscientes del patrón resultante en la investigación. Virtualmente cada elemento estilísticamente distintivo o funcionalmente significativo del registro arqueológico prehistórico tardío ha sido atribuido en algún momento, por algún investigador, a un origen oriental. En la Edad del Bronce, los paralelos propuestos consistían predominantemente en rasgos estilísticos bastante específicos que se concentraban en el Egeo y Anatolia. En el caso de la Edad del Cobre, el énfasis de la discusión se cambió hacia rasgos más funcionales y más amplios (metalurgia del cobre, enterramiento colectivo en tumbas de cámara, poblados fortificados con bastiones), aunque algunos paralelos particulares, bastante más extensamente distribuidos que para la Edad del Bronce, completarían la argumentación. En el caso del Neolítico, la llegada demostrable de domesticados de Próximo Oriente disminuyó, pero no eliminó totalmente, la necesidad de defender la importancia de la difusión oriental sobre bases estilísticas especiales. Diversos autores han discutido con energía los diferentes centros originarios propuestos y las rutas a través de las cuales las influencias se extendieron, pero prestaron relativamente poca atención a los mecanismos de su transmisión. Así, los excavadores de Los Millares o de Zambujal fueron muy conscientes de que aquellos yacimientos carecían de los rasgos concluyentemente coloniales de Ampurias o Toscanos, pero esto no les impidió denominar también colonias a sus yacimientos de la Edad del Cobre. Los colonos (o mercaderes o misioneros o prospectores) podían ser sugeridos como agentes difusores, pero, en el marco normativista, eran las propias ideas, no el carácter del agente por el cual las ideas eran transmitidas, las que eran de importancia primordial. Todos estaban de acuerdo en que el "contacto" era la clave del cambio cultural.

\section{II}

Este consenso fue barrido por Colin Renfrew, cuyo liderazgo ha sido el principal responsable del establecimiento de un enfoque procesualmente orientado para reemplazar al caótico normativismo que acabo de describir. El enfoque de Renfrew implicó diversas reformas de la práctica interpretativa previa. En primer lugar, los marcos cronológicos tuvieron que ser construidos primordialmente sobre la base de las determinaciones radiocarbónicas (Renfrew, 1973); las dataciones cruzadas tipológicas estiarían restringidas a semejanzas claramente documentadas y muy específicas entre regiones adyacentes. Esto evitaría la circularidad característica de los argumentos cronológicos previos, en los que la datación de las culturas arqueológicas prehistóricas descansaba sobre los contactos asumidos entre ellas, más que sobre correspondencias repetidas y detalladas entre sus respectivos inventarios. (En ausencia de cualquier datación absoluta excepto las históricas, la única esperanza de asignar fechas seguras a las culturas prehistóricas tardías del Mediterríneo central y occidental residía en asumir que eran posteriores al 3000 a. C.; había que encontrar objetos, y se encontraron, cuya inspiración oriental confirmara este marco). En segundo lugar, a falta de semejanzas estilísticas tan detalladas y complejas que pusieran de manifiesto un origen artesano común, el tráfico entre 
regiones tenía que establecerse por la atribución positiva de materias primas a fuentes particulares, por ejemplo mediante las señas de elementos traza distintivos (Renfrew et alii, 1965, 1968; Renfrew \& Peacey, 1968). En tercer lugar, la discusión sobre la naturaleza de dicho tráfico debía ser emprendida en términos de modelos explícitos, preferentemente cuantitativos que ofrecerían cierta esperanza de distinguir entre los diferentes tipos de intercambio (Renfrew, 1977). En ausencia de un volumen adecuado de bienes intercambiados, por supuesto, los problemas de muestreo obviarían modelos de ese tipo, y se asumiría la independencia regional.

El establecimiento de esas líneas directrices analíticas ha resultado en una reducción radical del alcance del "contacto" reconocido entre las regiones mediterráneas durante la Prehistoria. Habiendo sido excluidas las semejanzas generales y las coincidencias ocasionales que los normativistas habían usado para conectar regiones, cada área diferente ha llegado a ser considerada independiente en cuanto a su desarrollo. En la Península Ibérica, algo de marfil del Norte de Africa (Harrison \& Gilman, 1977) es importado en las Edades del Cobre y del Bronce, y dos fragmentos cerámicos micénicos han sido hallados en un contexto de la Edad del Bronce Final (Martín de la Cruz, 1988), pero no se ha encontrado un volumen sustancial de importaciones claramente documentadas del Mediterráneo oriental hasta bien avanzado el Primer Milenio a. C. En Italia, las importaciones claras más antiguas desde Grecia se datan en el s. XVI a. C., apareciendo un volumen significativo de comercio sólo en el s. XIV (Smith, 1987). Una doble cronología cruzada entre Creta y el Próximo Oriente no es posible con anterioridad al s. $X X$ a. C. Antes de esa época (Renfrew, 1972: 211-217), sólo tiene lugar un goteo de importaciones unidireccionales desde Egipto. Esas fechas indican claramente, por ejemplo, que una tecnología tan significativa como la metalurgia del cobre, la pieza central del difusionismo previo, se habría desarrollado independientemente en Iberia, Italia, y el Egeo. En cada una de esas áreas, la necesidad común de las élites emergentes del III milenio a. C. de concentrar, almacenar, y exhibir su riqueza habría llevado a una solución también común. Por supuesto, en la concepción moderna el intercambio no está excluido por completo como fuerza significativa en el desarrollo prehistórico tardío del Mediterráneo. Renfrew (1972) da gran importancia al "efecto multiplicador" que habría provocado la emulación competitiva de las élites en las regiones. La «interacción entre unidades políticas paritarias" ("peer polity interaction") (Renfrew \& Cherry, 1986) es concebida, sin embargo, como un fenómeno primordialmente intraregional. Las trayectorias evolutivas de cada región diferente se mantendrían sustancialmente independientes.

El autonomismo del punto de vista de los arqueólogos procesuales de la Prehistoria del Mediterráneo corre paralelo a la opinión dominante sobre el papel del comercio en época clásica, que destaca, en palabras de Keith Hopkins (1983: XI):

«la autosuficiencia celular de la economía antigua; cada granja, cada distrito, cada región cultivaba y hacia casi todo lo que necesitaba. La base principal de la riqueza era la agricultura... La escala del transporte interregional era muy pequeña. El transporte terrestre era demasiado caro, excepto para el acarreo de bienes de lujo. Incluso por mar, el comercio constituía solo una proporción muy pequeña de la producción bruta".

Andrew y Susan Sherratt (1991: 352) comentan que, «en los últimos años, esta actitud ha salido reforzada por efecto de la Nueva... Arqueología, que era especialmente hostil a las vagas "influencias" exteriores sobre asuntos tan básicos como la organización social». En mi opinión, el efecto sigue más bien otra dirección. Los historiadores sociales del Mundo Antiguo facilitaron a los procesualistas un terminus sub quo que los prehistoriadores sociales estaban obligados a respetar en sus reconstrucciones de épocas más antiguas y más sencillas. $\mathrm{Si}$ el comercio (es decir, un contacto arqueológiciamente documentable) era de escala reducida en época clásica, tenía que haber sido virtualmente insignificante en los períodos anteriores.

\section{III}

Inevitablemente, la nueva ortodoxia procesualista no ha demostrado ser convincente por completo. Los normativistas supervivientes, por 
supuesto, nunca han sido persuadidos: la revisión de las cronologías cortas requerida por la evidencia del radiocarbono simplemente ha provocado que los estudiosos de la vieja escuela reorientaran su búsqueda de paralelos a segmentos más antiguos de las secuencias del Mediterráneo oriental (p. e., Schüle, 1986). Muchos prehistoriadores que aceptan la verdad global del revisionismo procesualista han mantenido dudas bastante legítimas, sin embargo, sobre las versiones más radicales de autonomismo. Las élites emergentes de la Edad del Bronce en Europa ciertamente conservaron amplias redes de emulación y apoyo mutuo - la amplia distribución de estilos particulares de recipientes para beber y de armas refleja claramente esto-, y a veces esas redes parecen intersectarse con las esferas de sus equivalentes en el Mediterráneo oriental. Ciertamente algunos elementos de los inventarios de los enterramientos de las élites durante la Edad del Bronce europea tienen reminiscencias de sus análogos egeos. Como Kristian Kristiansen (1991: 27), a quien difícilmente puede considerarse un tradicionalista obstinado, indica:

"Los jefes supremos (2) controlaban un intercambio elitista a larga distancia de metales, bienes de prestigio, y conocimiento exótico conexo. La ascensión de las jefaturas en torno al 1500 a. C. estaba ligada a un complejo ideológico y militar de guerreros aristocráticos que se extendieron desde el área micénica y el Asia Menor por Europa central y Escandinavia. Estaba caracterizado por atributos principalmente novedosos que incluían carros de guerra, taburetes, navajas de afeitar, y pinzas".

No sería difícil imaginar la propuesta de un argumento similar para explicar las copas y diademas encontradas en las tumbas argáricas de una fecha algo más antigua. Sería injusto, en mi opinión, descartar tales reivindicaciones de la significación del Mediterráneo oriental para la Europa bárbara como meros ejemplos de la reincidencia idealista del postmodernismo.

Subsiste, sin embargo, la pregunta: ¿cómo edificar una construcción explicativa que de cuenta de tales similaridades aparentes sin retroceder simplemente a las antiguas oscuras

(2) «Paramount chiefs" en el original en inglés. "influencias" (ahora redefinidas no menos oscuramente como «inspiraciones")? Ciertamente podemos aceptar las observaciones de Mary Helms (1988) sobre «la autoridad del conocimiento lejano" para conferir distinción y legitimidad a los miembros de una élite, y como está demostrado que el ámbar báltico llega hasta el Mediterráneo, cabría esperar algún flujo de información en dirección contraria. Sin embargo, hasta que el volumen de intercambio no fuera suficiente para dejar un registro arqueológico sustancial, estaría injustificado suponer que existiera una interdependencia materialmente significativa. Los jefes en Europa central y septentrional, o en la Península Ibérica, pueden haber usado objetos esotéricos del Mediterráneo oriental para demostrar su superioridad al vulgo, pero dada una base material para tal superioridad, si los modelos foráneos particulares usados para justificarla hubieran estado ausentes, el ejercicio de su poder por la élite apenas se habría visto obstaculizado: otros símbolos y justificaciones podrían haber sido preparadas a partir de inspiraciones foráneas diferentes e igualmente ténues, o podrían haber sido totalmente inventadas. No se puede dar ningún peso procesual a lo que Manuel Fernández-Miranda (1991: 89) califica como "contactos de tipo diverso y más difícil de detectar [que] ocurre[n] a lo largo... de la Edad del Bronce».

\section{IV}

En la prehistoria mediterránea el esfuerzo más prometedor para situar el contacto en un contexto procesual ha sido la aplicación de la teoría de los sistemas mundiales o del centr()periferia. La visión general de Gordon Childe de la relación entre el Próximo Oriente y Europa durante la época prehistórica fue, por supuesto, una primera versión del enfoque de los sistemas mundiales. Childe era muy consciente, sin enbargo, de que la explicación que proponía del desarrollo de la Edad del Cobre y la Edad del Bronce europeas estaba más sostenida por su lógica y coherencia internas que por una evidencia real. El podía argumentar que «los poblados costeros, representados por los cementerios [de tumbas de cámara], habían sido establecidos principalmente allí donde las colo- 
nias griegas en Occidente fueron realmente situadas en época histórica; así el cementerio de Paestum podía realmente pertenecer al precursor prehistórico de Cumae..., aquél cercano a Arles al precursor de Marsella" (Childe, 1958: 117), pero inmediatamente admitía que, "si los prospectores y mercaderes del Egeo ayudaron a fundar las colonias de la Edad del Bronce [en el Mediterráneo occidental], no llevaron con ellos un equipamiento material e ideológico completo, ni mantuvieron unos contactos con sus patrias que les mantuvieran abastecidos con sus manufacturas como hicieron los colonos griegos históricos" (Ibid.: 177-178). Childe presumiblemente encontró su escenario explicativo global lo suficientemente convincente como para que la casi completa ausencia de confirmación empírica pudiera ser excusada. En general, Childe no estaba muy preocupado por los problemas de medir las variables sociales manejando evidencia arqueológica, pero el efecto de esta negligencia fue hacer indistinguible, en lo que a los hechos se refiere, su teoría esencialmente procesual de las relaciones entre el Próximo Oriente y Europa, de una explicación difusionista, normativista de los mismos fenómenos. Cuando a los conjuntos de similaridades se les concedía el mismo peso como evidencia que a las importaciones, las ideas adoptadas del exterior, y no las interacciones sociales que dirigian la transmisión, eran claramente las que provocaban el cambio cultural: los bárbaros adoptarían elementos culturales de sus vecinos civilizados porque aquellos elementos eran, de algún modo, intrínsecamente mejores. Las teorías de sistemas mundiales que no especifican los procesos que ligan el centro y la periferia (p. e., Frank, 1993), todavía ahora, pueden generar una incoherencia y especulación dignas de los peores excesos del normativismo tradicional.

Incluso en el caso de la Edad del Bronce egea, donde la existencia de intercambio con el Próximo Oriente era demostrable manejando los criterios más exigentes, la ausencia de una explicación precisa de cómo se organizaba ese intercambio hacia posible que los procesualistas (p. e., Halstead \& O'Shea, 1982) construyeran un escenario autonomista para la aparición de los palacios minoicos y micénicos. Estos serían centros de redistribución interna y de almacenamiento social que organizarían y garantizarian un sistema económico intensificado e internamente especializado. El comercio exterior a larga distancia sería una actividad consistente en «intercambios no documentados que tienen lugar sobre una base irregular y oportunista, a través de la cual artículos artesanales... fueron exportados como "regalos de huésped" como devolución por materias primas" (Halstead, 1988: 526), en el mejor de los casos, un asunto de importancia secundaria.

En el caso del Egeo, este punto de vista ya no puede sostenerse. Primero, tenemos análisis detallados, basados en textos, de las operaciones de las economías palaciegas en la Edad del Bronce del Próximo Oriente. Como Mario Liverani (1987: 69) indica, el «sistema de producción monopolística y de comercio interregional intensivo estaba ligado al sistema político... con contactos llevados a cabo al más alto nivel político", principalmente el palacio. Era un sistema en el que los artesanos y mercaderes operaban como especialistas vinculados (Brumfiel \& Earle, 1987) al servicio de los monarcas que trataban con unos y otros en términos de "reciprocidad y hermandad" (Liverani, 1987: 67). En segundo lugar, tenemos la evidencia de los naufragios de la Edad del Bronce en el Cabo Gelidonya y Ulu Burun (Bass, 1991). Como Anthony Snodgrass (1991: 18) comenta, aquellos barcos sólo pudieron haber tenido como destino un palacio: el cobre y el estaño de la carga del de Ulu Burun "probablemente habrían facilitado el bronce suficiente para equipar todo el ejército de un rey micénico"; supera cinco veces la cantidad de bronce citada en las tabletas de Pilos, y estaba acompañada de defensas de elefante, huevos de avestruz, plata, oro, y vasijas de faienza, los regalos personales que habrían acompañado los intercambios entre los hermanos reales. En tercer lugar, contamos con la evidencia arqueológica y textual de los propios palacios egeos. Esta evidencia deja claro que éstos eran réplicas a pequeña escala de sus equivalentes en el Próximo Oriente. Los palacios controlaban las operaciones agrícolas que estaban orientadas hacia la producción de bienes de lujo (vino, lana para tejidos, aceite de oliva para perfume) y disponían de los servicios de especialistas vinculados, dedicados a esa producción. Controlaban también la producción del metal recibido a través de los intercambios de regalos 
entre las élites. Los únicos datos que nos faltan son las cartas dirigidas por los gobernantes de los palacios egeos a sus equivalentes egipcios o próximo-orientales. Todos los rasgos que dan a la Edad del Bronce tardía del Egeo su carácter "complejo" son rasgos asociados específicamente con los palacios, cuyos gobernantes fueron capaces de aumentar grandemente los excedentes que podian obtener por tributos en especie comprometiéndose en intercambios de riqueza con sus equivalentes próximo-orientales más poderosos. Los palacios eran tan completamente próximo-orientales que, cuando el sistema de intercambio de riqueza reventó como una burbuja y los palacios desaparecieron, no quedó memoria de ellos en las tradiciones históricas griegas.

\section{V}

El éxito de un enfoque de centro-periferia para explicar gran parte de lo que caracteriza la Edad del Bronce minoico-micénica puede servir para mostrarnos lo que está faltando en otros casos donde se aplica una jerga de sistemas mundiales. Como mínimo, la influencia del centro sobre la periferia tiene que ser lo bastante fuerte para alterar sustancialmente el curso de la historia de la periferia. El contacto con el Próximo Oriente provocó en las élites egeas más que un simple cambio en sus patrones de consumo y ostentación: alteró de manera importante sus estrategias fundamentales de explotación (Gilman, 1991). En contraste, cuando los micénicos se implicaron comercialmente con el Mediterráneo central, las instituciones sociales nativas no cambiaron significativamente. En Cerdeña, por ejemplo, la trayectoria nurágica no fue sustancialmente alterada ni cuando las importaciones micénicas aparecieron, ni cuando desaparecieron (Webster, 1991). Más allá, la Edad del Bronce egea podía haber facilitado (mediante mecanismos que son imposibles de especificar) la inspiración para algunos accesorios de las élites, pero el desarrollo de la Edad del Bronce en la Europa bárbara fue esencialmente independiente del Egeo: cuando el sistema palacial se colapsó, apenas hubo diferencia. La Europa bárbara no era el Tercer Mundo del Egeo; no era periférico, sino externo.
Del mismo modo es dificil interpretar los florecimientos del inicio de la Edad del Hierro que he citado al comienzo de este artículo como fenómenos dependientes de los estímulos de centros orientales más desarrollados. En Grecia e Italia, al menos, aspectos críticos de la transformación de la Edad del Hierro estaban en pleno funcionamiento antes de las fases totalmente orientalizantes de las secuencias durante los siglos VII e inicio del VI. Esos aspectos incluyen:

1. La metalurgia del hierro se generaliza. La mayor disponibilidad y menor coste de instrumentos cortantes eficaces habría incrementado la productividad tecnológica en todos los aspectos, desde la agricultura a la producción artesanal. Como Childe (1942: 183) resaltaba, el hierro "democratizó la agricultura, la industria y también la guerra". Igualmente importante fue que el hierro "subvirtió los sistemas [de comercio] basados en la adquisición y circulación de bronce... También hizo que el control de los metales preciosos fuera incluso más importante" (Sherratt \& Sherratt, 1993: 362). En la Edad del Bronce, la aplicación de la metalurgia a necesidades prácticas había sido frenada por la función central del metal, controlado por la élite, como medio de concentrar y exhibir riqueza. En la Edad del Hierro, la metalurgia práctica quedó desligada de la riqueza, con amplias consecuencias tecnológicas.

2. Quizá la más significativa de esas consecuencias fuera el desarrollo de una agricultura esencialmente moderna. En Grecia, por ejemplo, el cultivo del olivo sólo comienza a alcanzar su importancia actual durante la Edad Oscura postmicénica (Runnels \& Hansen, 1986). En Italia, la completa implantación del policultivo mediterráneo probablemente empieza en la Edad del Hierro inicial (Barker, 1985: 82).

3. Tanto fuertes incrementos en las densidades de población, como el establecimiento cle verdaderas ciudades parecen ya haber iniciado su desarrollo posterior en torno al s. VIII en Grecia (Snodgrass, 1980) y Etruria (Potter, 1979).

4. La evidencia funeraria (Morris, 1987) sugiere con fuerza que las instituciones políticas características y las divisiones en clases sociales 
de la ciudad estado clásica tienen sus raíces en las fases iniciales de la Edad del Hierro de Grecia.

Sin duda, los contactos con los mercaderes del Próximo Oriente tuvieron lugar en Grecia durante todo el Protogeométrico y el Geométrico, pero, como Ian Morris (1987: 203) indica, "ciertamente nada sugiere que la Grecia del s. VIII deba ser vista como un área periférica de un sistema mundial». Casi cabe decir otro tanto de la Italia Protovillanoviana y Villanoviana. La evidencia indica que en Grecia, como en Italia, los mercaderes orientales establecieron sus operaciones en las áreas donde los desarrollos sociales y económicos pre-existentes habían creado una demanda para sus ofertas.

En España, el tema es mucho menos claro. Por un lado, una escuela de pensamiento ha visto el desarrollo ibérico como el resultado de influencias fenicias sobre una población indígena relativamente sin evolucionar. Carlos González Wagner proporciona una lúcida explicación de cómo serían caracterizados de acuerdo con ella los grupos del final de la Edad del Bronce en la Iberia meridional:

«una forma doméstica de producción... encaminada más al aprovisionamiento que al comercio lucrativo, que funciona en un estado de anarquía primitiva, debidamente corregida por los vínculos de parentesco... y la reciprocidad que éstos suponen" (González Wagner, 1983: 9).

Estas tribus o pequeñas jefaturas se verían estimuladas hacia la empresa comercial y el rápido cambio social por la introducción fenicia de la metalurgia avanzada. Un punto de vista similar es propuesto por Richard Harrison que señala "la pobreza tecnológica», "la clara falta de riqueza acumulada para atraer a los comerciantes lejanos", "las densidades de población relativamente bajas, las pequeñas aldeas normalmente sin defensa" como evidencia de que "Iberia al final de la Edad del Bronce no era El Dorado" (Harrison, 1988: 40). Subyacente a estas opiniones está la noción de que El Argar es sucedido por una "edad oscura" de involución hacia condiciones económicas más simples y sociales menos jerárquicas (cf. Lull, 1983: 456457).

Por otro lado están quienes sitúan las raíces de la expansión del inicio de la Edad del Hierro en la red comercial indígena de la Edad del Bronce Atlántica. La evidencia firme del comercio fenicio en Iberia se fecha en el s. VIII (Aubet Semmler, 1986), en tanto que una red de intercambio que conectaba Andalucía con el Mediterráneo central habría estado funcionando al menos un siglo antes (Ruiz-Gálvez, 1986; Fernández-Miranda, 1991). Desde esta perspectiva, pues:

«El comercio y la aculturación fenicia actuaron sobre [una] compleja estructura socioeconómica, caracterizada por la rivalidad en el interior de las élites por alcanzar mayores cotas de prestigio...Los fenicios se aprovecharon de ese peculiar contexto, sin que llegaran a crear un orden económico nuevo" (Barceló, 1992: 269).

Implícita en esta opinión está una visión cle la economía y la sociedad de la Edad del Bronce post-argárica completamente diferente. Una agricultura más productiva, una industria metalúrgica más sofisticada y a mayor escala, y un sistema más estable de jerarquía social (ya no debilitada por el persistente conflicto entre $\mathrm{co}$ munidades característica de la Edad del Bronce más antigua) serían los requisitos previos al establecimiento de la red de intercambio atlántico (cf. Lull et alii, 1992: 247-249).

Los paralelos entre el caso ibérico y sus análogos italianos y griegos pueden dar algún fundamento a la segunda de esas versiones, pero la evidencia sobre la organización económica y social que fundamentaría adecuadamente una u otra todavía tiene que ser desarrollada. El impacto de los contactos fenicios y griegos durante el Primer Milenio en Iberia sólo puede proponerse en un ámbito que dé primacia explicativa al marco político y económico del intercambio. Se ha hecho un trabajo sólido para lograr una comprensión empíricamente fundiamentada de la economía política del núcleo del sistema mundial mediterráneo (p. e., Frankenstein, 1979): aquél trabajo todavía tiene que ser emprendido para la periferia mediterránea occidental. 


\section{BIBLIOGRAFIA}

Aubet Semmi.er, M. E. (1986): “Los fenicios en España: estado de la cuestión y perspectivas». En G. del Olmo Lete \& M. E. Aubet Semmler (eds.): "Los fenicios en la Península Ibéricam, 1. Editorial Ausa, Sabadell: 9-38.

BARCELó, J. A. (1992): “Una interpretación del Bronce final en el sudoeste de la Península Ibérica". Trabajos de Prehistoria, 49: 259-275.

BAss, G. (1991): "Evidence of trade from Bronze Age shipwrecks". En N. H. Gale (ed.): "Bronze Age trade in the Mediterraneann. Paul Åströms Verlag. Jensered: 6982.

BRUMFIEL, E. M. y EARLE, T. K. (1987): "Specialization, exchange, and complex societies: an introduction". En E. M. Brumfiel \& T. K. Earle (eds.): "Specialization, exchange, and complex societies". Cambridge University Press, Cambridge: $1-9$.

CHILDE, V. G. (1942): “What happened in history». Penguin, Harmondsworth.

- (1958): «The prehistory of European society». Penguin. Harmondsworth.

FERNÁNDEZ-MIRANDA, M. (1991): "Tartessos: indígenas, fenicios y griegos en Huelva". Atti del II Congresso Internazionale di Studi Fenici e Punici, 1: 87-96. Roma.

Frank, A. G. (1993): “The Bronze Age world system and its cycles". Current Anthropology, en prensa.

FRANKenstein, S. (1979): "The Phoenicians in the far west: a function of neo-Assyrian imperialism». En M. T. Larsen (ed.): «Power and propaganda: a symposium on ancient empiresn. Akademisk Forlag. Copenhague: 263294.

GiLman, A. (1991): “Trajectories towards social complexity in the later prehistory of the Mediterranean". En T. Earle (ed.): "Chiefdoms: power, economy, and ideology". Cambridge University Press. Cambridge: 146-168.

González Marcén, P.; Lull, V. y Risch, R. (1992): "Arqueología de Europa, 2250-1200 A.C.: una introducción a la "edad del bronce"». Editorial Síntesis. Madrid.

GonZÁlez WAGNeR, C. (1983): “Aproximación al proceso histórico de Tartessos". Archivo Español de Arqueología, LVI: 3-36.

HALSTEAD, P. (1988): "On redistribution and the origin of Minoan-Mycenaean palatial Economies». En E. B. French \& K. A. Wardle (eds.): "Problems in Greek prehistory". Bristol Classical Press. Bristol: 519-529.

Halstead, P. y O'SheA, J. (1982): “A friend in need is a friend indeed: social storage and the origins of social ranking". En C. Renfrew \& S. Shennan (eds.): "Ranking, resource and exchange: aspects of the archaeology of early European society". Cambridge University Press. Cambridge: $92-99$.

HARRISON, R. J. (1988): "Spain at the dawn of history". Thames and Hudson. Londres.

HARRISON, R. J. y GILMAN, A. (1977): "Trade in the second and third millennia B.C. between the Maghreb and Iberia». En V. Markotic (ed.): “Ancient Europe and the Mediterranean: studies in honour of Hugh $O$. Hencken". Aris \& Phillips, Warminster, Wilts.: 90-104.

HeLms, M. (1988): «Ulysses' sail: an ethnographic odyssey of power, knowledge, and geographical distance». Princeton University Press. Princeton, NJ.

HoPKINS, K. (1983): “Introduction". En P. Garnsey, K. Hopkins \& C. R. Whittaker (eds.): "Trade in the ancient economy". University of California Press. Berkeley: ixxxv.

KRISTIANSEN, K. (1991): "Chiefdoms, states, and systems of social evolution". En T. Earle (ed.): "Chiefdoms: power, economy and ideology". Cambridge University Press. Cambridge: $16-43$.

LiVERANI, M. (1987): "The collapse of the Near Eastern regional system at the end of the Bronze Agen. En M. Rowlands; M. Larsen \& K. Kristiansen (eds.): “Centre and periphery in the ancient world". Cambridge University Press. Cambridge: 66-73.

Lull, V. (1983): “La "cultura” de El Argar». Akal Editor. Madrid.

MaRTín de la CRUz, J. C. (1988): “Mykenische Keramik aus bronzezeitlichen Siedlungsschichten von Montoro am Guadalquivir". Madrider Mitteilungen, XXIX: 77. 92.

MoRris, I. (1987): "Burial and ancient society: the rise of the Greek city-state». Cambridge University Press. Carnbridge.

RENFREW, C. (1972): "The emergence of civilisation: the Cyclades and the Aegean in the third millennium B.C." Methuen. London.

- (1973): "Before civilization: the radiocarbon revolution and prehistoric Europen. Jonathan Cape. Londres.

- (1977): "Alternative models for exchange and spatial distribution». En T. K. Earle \& J. E. Ericson (eds.): "Exchange systems in prehistory". Academic Press. Nueva York: 71-90.

Renfrew, C.; J. R. CANn y Dixon, J. E. (1965): “Obsidian in the Aegean". Annual of the British School of Archaeology at Athens, LX: 225-247.

Renfrew, C. y Cherry, J. (eds.) (1986): "Peer polity interaction and socio-political change». Cambridge University Press. Cambridge.

Renfrew, C.; Dixon, J. E. y CANN, J. R. (1968): “Further analysis of Near Eastern obsidian". Proceedings of the Prehistoric Society, XXXIV: 319-331.

RENFREW, C. y PEACEY, J. E. (1968): “Aegean marble: a petrological study". Annual of the British School of Archaeology at Athens, LXIII: 45-66.

Ruiz-GÁlVEZ, M. L. (1986): “Navegación y comercio entre el Atlántico y el Mediterráneo a fines de la Edad del Bronce". Trabajos de Prehistoria, XLIII: 9-42.

Runnels, C. N. y J. Hansen (1986): "The olive in the prehistoric Aegean: the evidence for domestication in the early Bronze Agem. Oxford Journal of Archaeology, V: $299-308$.

Shule, W. (1986): "El cerro de la Virgen de la Cabeza, Orce-Granada: consideraciones sobre su marco ecológico y cultural". Homenaje a Luis Siret (1934-1984). Consejería de Cultura de la Junta de Andalucía. Sevilla.

SherRatT, A. y SherratT, S. (1991): "From luxuries to commodities: the nature of Mediterranean Bronze Age trading systems". En N. H. Gale (ed.): "Bronze Age trade in the Mediterranean". Paul Aströms Verlag. Jensered: 351-385.

SherRatT, S. y SHERratT, A. (1993): "The growth of the 
Mediterranean economy in the early first millennium B.C.". World Archaeology, XXIV: 361-378.

SMITH, T. R. (1987): Mycenaean trade and interaction in the west central Mediterranean, 1600-1000 B.C. BAR International Series 371, Oxford.

SNODGRASS, A. M. (1991): "Bronze Age exchange: a minimalist position». En N. H. Gale (ed.): “Bronze Age trade in the Mediterranean". Paul Åströms Verlag. Jensered: 1520.

WEBSTER, G. S. (1991): "The functions and social significance of nuraghi: a provisional modeln. En B. S. Frizell (ed.): "Arte militare e architettura nuragica". Skrifter Utgivna av Svenska Institutet i Rom, 4․, XLVIII, Estocolmo: 169-185. 